This paper is available online at http://www.math.nthu.edu.tw/tjm/

\title{
INTEGRAL RICCI CURVATURES, VOLUME COMPARISON AND FUNDAMENTAL GROUPS OF COMPACT RIEMANNIAN MANIFOLDS
}

\author{
Seong-Hun Paeng
}

\begin{abstract}
We obtain a relative volume comparison estimate in the universal covering space under bounds on the integral Ricci curvature and the weak $C^{1}$-norm of metric. From this volume comparison, we obtain similar results on the fundamental group as in $[1,7,8]$.
\end{abstract}

\section{INTRODUCTION}

The Bishop-Gromov relative volume comparison theorem is one of the most important tools to study global structures of Riemannian manifolds with Ricci curvatures bounded below. From the volume comparison in the universal covering space of an $n$-dimensional compact manifold $M$ with the Ricci curvature $\operatorname{Ric}_{M} \geq \lambda$, we have several results about the fundamental group of $M$. In [1], Anderson proved that there are finitely many isomorphism classes of fundamental groups of compact manifolds with Ricci curvatures and volumes bounded below. Using this theorem, Wei [7] proved that almost nonnegative Ricci curvature and a lower bound on the volume implies that $\pi_{1}(M)$ is of polynomial growth with rank $\leq n$. Under the same conditions as [7], Yun [8] showed that $\pi_{1}(M)$ is almost abelian.

There are many attempts to replace pointwise curvature conditions with integral curvature conditions. In [4], Petersen and Wei obtained a Bishop-Gromov type volume comparison theorem with an integral Ricci curvature bound. But the volume comparison estimate in [4] is not applicable to the balls in the universal covering space $\tilde{M}$ only with the assumption on the integral Ricci curvature bound of $M$ (see

Received August 29, 2005, accepted December 1, 2005.

Communicated by Shu-Cheng Chang.

2000 Mathematics Subject Classification: Primary 53C20, secondary 53C23.

Key words and phrases: Integral Ricci curvature, Volume comparison.

The work was supported by Graint No. R01-2006-000-10047-0(2006) from the Basic Researc Program

of the Korea Science \& Engineering Foundation. 
Section 2). For the proof of the theorems about the fundamental group such as in $[1,7,8]$, the volume comparison in the universal covering space is essential.

We can consider some additional assumptions for the volume comparison in the universal covering space. For example, if we assume additionally that the volume of $M$ satisfies that $\operatorname{vol}(M) \geq v>0$ and $\int_{M}\|R\|^{p} \geq \Omega$ for the curvature tensor $R$, then we can obtain the volume comparison in the universal covering space by $C^{0, \alpha}$-compactness theorem. (For the precise reason, see Section 2.) But we cannot obtain explicit values in Theorem 1.1 and Theorem 1.2 if we use $C^{0, \alpha}$-compactness theorem. More generally, we can obtain the volume comparison in the universal covering space under the additional condition that the weak harmonic $C^{0, \alpha}\left(L^{1, p}\right)$ norm $\|(M, g)\|_{C^{0}, \alpha, r}^{W, h} \leq Q(r)$ for a function $Q$ satisfying $\lim _{r \rightarrow 0} Q(r)=0$ in [6]. (For the precise definition of the norm, see section 2 or [6].) Recall that the weak (harmonic) norm is suggested as a generalization of the curvature in [6] and if the weak harmonic norm $\|(M, g)\|_{C^{0}, \alpha, r}^{W, h} \leq Q(r)$ for a function $Q$ satisfying $\lim _{r \rightarrow 0} Q(r)=0$, then the metric $g$ can be smoothed to a metric $\tilde{g}$ satisfying $\left|K_{\tilde{g}}\right| \leq k$ for some constant $k$ depending on $Q$, where $K_{\tilde{g}}$ is the the sectional curvature of $\tilde{g}[6]$.

In this paper, we assume that $\operatorname{vol}(M) \geq v$ and the weak $C^{1}$-norm $\|(M, g)$ $\| C_{C^{1}, r_{0}}^{W} \leq k$ for some constant $k$ to obtain an explicit relative volume comparison in the universal covering space $\tilde{M}$, where a lower bound on the volume is essential condition in $[1,7,8]$. Although we use a weak $C^{1}$-norm bound instead of a $C^{0, \alpha_{-}}$ norm bound, our assumption on the weak norm is much simpler than that of [6] in the sense that we do not assume that the weak norm is bounded by a function $Q$ such that $\lim _{r \rightarrow 0} Q(r)=0$ and do not use any harmonicity assumption. Hence the metrics satisfying our conditions are not smoothed to metrics with a bounded sectional curvature.

First, we define the following notations about the integral Ricci curvature: Let $g(x)$ be the smallest eigenvalue of the Ricci tensor at $x \in M$ and $u_{+}=\max (0, u)$ is the positive part of $u$. For $2 p>n$ and $\lambda \leq 0$,

$$
\begin{aligned}
k(\lambda, p) & =\int_{M}\left((-g(x)+(n-1) \lambda)_{+}\right)^{p} d v, \\
\bar{k}(\lambda, p) & =\frac{k(\lambda, p)}{\operatorname{vol}(M)}, \\
k_{x}(\lambda, p, R) & =\int_{B(x, R)}\left((-g(x)+(n-1) \lambda)_{+}\right)^{p} d v \text { for } x \in \tilde{M} .
\end{aligned}
$$

If $\operatorname{Ric}_{M} \geq \lambda$, then $k(\lambda, p)=0$. Let $v_{\lambda}(n, r)$ be the volume of $r$-ball in the $n$ dimensional simply connected manifold with constant curvature $\lambda$. We obtain the following volume comparison in $\tilde{M}$ with an integral Ricci curvature bound: 
Theorem 1.1. Let $M$ be an $n$-dimensional compact Riemannian manifold and $x$ be a point in $\tilde{M}$. For given $r_{0}, v, D, \lambda, R>0$ and $p>n / 2$, we can find constants $K_{r_{0}}>0$ and $C\left(n, p, \lambda, R, v, D, r_{0}\right)>0$ explicitly such that if $\operatorname{diam}(M) \leq D$, $\operatorname{vol}(M) \geq v$ and $\|(M, g)\|_{C^{1}, r_{0}}^{W} \leq K_{r_{0}}$, then

$$
\left(\frac{\operatorname{vol}(B(x, R))}{v_{\lambda}(n, R)}\right)^{\frac{1}{2 p}}-\left(\frac{\operatorname{vol}(B(x, r))}{v_{\lambda}(n, r)}\right)^{\frac{1}{2 p}} \leq C\left(n, p, \lambda, R, v, D, r_{0}\right) k(\lambda, p)^{\frac{1}{2 p}}
$$

for $r<R$, where $B(x, R)$ is the R-ball centered at $x \in \tilde{M}$. When $r=0$, we obtain that

$$
\operatorname{vol}(B(x, R)) \leq\left(1+C\left(n, p, \lambda, R, v, D, r_{0}\right) k(\lambda, p)^{\frac{1}{2 p}}\right)^{2 p} v_{\lambda}(n, R) .
$$

We will take $K_{r_{0}}$ such that $K_{r_{0}} \leq 1 / 100$ and $K_{r_{0}} / r_{0} \leq 1 / 100$. Note that $\lim _{r_{0} \rightarrow 0}\|(M, g)\|_{C^{1}, r_{0}}^{W}=0$ for a fixed manifold $M$, which implies that we can find $r_{0}$ for a fixed $M$ such that $\|(M, g)\|_{C^{1}, r_{0}}^{W} \leq K_{r_{0}}$. The above theorem is about the class of manifolds with a uniform lower bound on the scale $r_{0}$ for which the weak $C^{1}$-norm is sufficiently small. Then we can prove Anderson [1] and Yun [8]'s results on the fundamental group:

Theorem 1.2. Let $r_{0}, C_{0}, D, v$ be positive constants and $p>n / 2$. We denote by $\mathcal{M}\left(r_{0}, D, v\right)$ the class of compact n-manifolds which satisfy that

$$
\begin{aligned}
\|(M, g)\|_{C^{1}, r_{0}}^{W} & \leq K_{r_{0}}, \\
\operatorname{diam}(M) & \leq D \\
\operatorname{vol}(M) & \geq v
\end{aligned}
$$

where $K_{r_{0}}$ is the constant in Theorem 1.1.

(a) There exists an $\epsilon\left(n, p, \lambda, v, D, r_{0}\right)>0$ such that if $k(\lambda, p)<\epsilon$, there are only finitely many isomorphism classes of $\pi_{1}(M)$ for $M \in \mathcal{M}\left(r_{0}, D, v\right)$.

(b) There exists an $\tilde{\epsilon}\left(n, p, v, D, r_{0}\right)>0$ such that if $k(0, p)<\tilde{\epsilon}$, then the fundamental group of $M \in \mathcal{M}\left(r_{0}, D, v\right)$ is almost abelian.

We can obtain an upper bound on the number of isomorphism classes of $\pi_{1}(M)$ and $\epsilon, \tilde{\epsilon}$ explicitly. As a corollary of Theorem 1.1, we obtain the following corollary for an arbitrary $C^{1, \alpha}$-norm bound $k_{0}$ :

Corollary 1.3. Let $M$ be an n-dimensional compact Riemannian manifold and $x$ be a point in $\tilde{M}$. For given $\lambda, R, v, D, r_{0}, k_{0}>0$ and $p>n / 2$, we can 
find constants $C_{1}\left(n, p, \lambda, R, v, D, r_{0}, k_{0}\right)>0$ explicitly such that if $\operatorname{diam}(M) \leq D$, $\operatorname{vol}(M) \geq v$ and $\|(M, g)\|_{C^{1, \alpha}, r_{0}}^{W} \leq k_{0}$, then

$$
\left(\frac{\operatorname{vol}(B(x, R))}{v_{\lambda}(n, R)}\right)^{1 / 2 p}-\left(\frac{\operatorname{vol}(B(x, r))}{v_{\lambda}(n, r)}\right)^{\frac{1}{2 p}} \leq C_{1}\left(n, p, \lambda, R, v, D, r_{0}, k_{0}\right) k(\lambda, p)^{\frac{1}{2 p}}
$$

for $r<R$ and $x \in \tilde{M}$. When $r=0$, we obtain that

$$
\operatorname{vol}(B(x, R)) \leq\left(1+C_{1}\left(n, p, \lambda, R, v, D, r_{0}, k_{0}\right) k(\lambda, p)^{\frac{1}{2 p}}\right)^{2 p} v_{\lambda}(n, R) .
$$

Instead of $\|(M, g)\|_{C^{1, \alpha}, r_{0}}^{W} \leq k_{0}$, we can use the weak $L^{2, p}$-norm $\|(M, g)\|_{L^{2, p}, r_{0}}^{W} \leq$ $k_{0}$. We can obtain Theorem 1.2 under an arbitrary weak $C^{1, \alpha}$-norm bound $k_{0}>0$.

\section{PReLIMINARIES}

In this section, we review the definition of the (weak) norm defined in [6] and observe what is the obstruction for the volume comparison in $\tilde{M}$. First, the definition of $C^{k, \alpha}$-norm defined in [6] is as follows:

Definition 2.1. The $C^{k, \alpha}$-norm of $(M, g)$ on scale $r_{0}>0,\|(M, g)\|_{C^{k, \alpha}, r_{0}}$ is defined to be the infimum of positive number $Q$ such that there exists embeddings

$$
\phi_{\tau}: B\left(0, r_{0}\right) \subset \mathbb{R}^{n} \rightarrow U_{\tau} \subset M
$$

with the following properties:

1. $e^{-2 Q} \delta \leq g_{\tau} \leq e^{2 Q} \delta$,

2. Every metric ball $B\left(p, \frac{r}{10} e^{-Q}\right)$ lies in some $U_{\tau}$,

3. $r_{0}^{|l|+\alpha}|| \partial^{l} g_{\tau, i j} \|_{C^{0, \alpha}} \leq Q$ for all multi-indices $l$ with $0 \leq|l| \leq k$, where $g_{\tau}=\phi_{\tau}^{*} g$.

The weak norm $\|(M, g)\|_{C^{k, \alpha}, r}^{W}$ is defined in a similar way except that $\phi_{\tau}$ is assumed to be a local diffeomorphism. If we require that $\phi_{\tau}$ 's are harmonic, then we call it the harmonic norm. They obtained a generalized almost flat manifold and a bound on Betti number under the condition on the weak harmonic norm stated in Section 1 instead of curvature [6]. But in this paper we do not need to consider the harmonic norm.

Now we observe why we cannot apply the volume comparison theorem in [4] to the universal covering space directly. We denote the function $\left((-g(x)+(n-1) \lambda)_{+}\right)$ by $\rho(x)$. By [4], we have the following volume comparison theorem: 
Theorem 2.2. Let $M$ be a Riemannian manifold and $x$ be a point in $M$. Then there exists a constant $C(n, p, \lambda, R)$ which is nondecreasing in $R$ such that when $r<R$, we have

$$
\left(\frac{\operatorname{vol}(B(x, R))}{v_{\lambda}(n, R)}\right)^{1 / 2 p}-\left(\frac{\operatorname{vol}(B(x, r))}{v_{\lambda}(n, r)}\right)^{1 / 2 p} \leq C(n, p, \lambda, R) k_{x}(\lambda, p, R)^{1 / 2 p} .
$$

When $r=0$, we obtain that

$$
\operatorname{vol}(B(x, R)) \leq\left(1+C(n, p, \lambda, R) k_{x}(\lambda, p, R)^{1 / 2 p}\right)^{2 p} v(n, \lambda, R) .
$$

As a corollary in [4], for any $\beta<1$, there exists an $\epsilon(n, p, \lambda, \beta, R)>0$ such that if

$$
\frac{k_{x}(\lambda, p, R)}{\operatorname{vol}(B(x, R))}<\epsilon
$$

then for $r<R$,

$$
\beta \frac{v_{\lambda}(n, r)}{v_{\lambda}(n, R)} \leq \frac{\operatorname{vol}(B(x, r))}{\operatorname{vol}(B(x, R))}
$$

Hence if we obtain (2.1) in the universal covering space $\tilde{M}$, we can prove our theorems by the same arguments as in $[1,7]$. But $(2.1)$ is not clear in the universal covering space even if $\bar{k}(\lambda, p)$ is very small. Precisely, if $\bigcup_{i=1}^{N} F_{i}$ is the minimal union of fundamental domains to cover $B(x, R)$, then

$$
\begin{aligned}
\frac{k_{x}(\lambda, p, R)}{\operatorname{vol}(B(x, R))} & =\frac{1}{\operatorname{vol}(B(x, R))} \int_{B(x, R)} \rho(x)^{p} d v \\
& \leq \frac{\operatorname{vol}\left(\bigcup_{i} F_{i}\right)}{\operatorname{vol}(B(x, R))} \frac{1}{\operatorname{vol}\left(\bigcup_{i} F_{i}\right)} \int_{\cup_{i} F_{i}} \rho(x)^{p} d v \\
& \leq \frac{\operatorname{vol}(B(x, R+2 D))}{\operatorname{vol}(B(x, R))} k(\lambda, p) .
\end{aligned}
$$

If we have a bound on $\frac{\operatorname{vol}(B(x, R+2 D))}{\operatorname{vol}(B(x, R))}\left(\right.$ e.g. $\left.\operatorname{Ric}_{M} \geq \lambda\right)$, we can apply the volume comparison of [4] to the universal covering space. But in our case, it is not clear.

One of the simplest conditions to obtain a bound on $\frac{\operatorname{vol}(B(x, R+2 D))}{\operatorname{vol}(B(x, R))}$ is $\operatorname{Ric}_{M} \geq \lambda$ [9]. But it seems to be too strong assumption. Another condition is $\operatorname{vol}(M) \geq v>0$ and $\int_{M}\|R\|^{p} \geq \Omega$, which implies $C^{0, \alpha}$-compactness [6]. Then the metric can be smoothed to $\tilde{g}$ slightly by [6], so that $\left|K_{\tilde{g}}\right| \leq k$ for some constant $k$. Hence we have 
a bound for $\frac{\operatorname{vol}(B(x, R+2 D))}{\operatorname{vol}(B(x, R))}$. But we cannot obtain an explicit volume comparison in the universal covering space with $C^{0, \alpha}$-compactness theorem. For example, $\epsilon$ in (2.1) cannot be obtained. More generally, we can obtain the volume comparison for the universal covering space as in [4] with $\|(M, g)\|_{C^{0, \alpha}, r}^{W, h} \leq Q(r)$ for a function $Q$ satisfying $\lim _{r \rightarrow 0} Q(r)=0$ as stated in Section 1 since the metric $g$ also can be smoothed to a metric $\tilde{g}$ satisfying $\left|K_{\tilde{g}}\right| \leq k$ for some constant $k$ which depends on $Q$ [6].

Our main purpose is to obtain an explicit volume comparison in the universal covering space with a constant bound on the weak $C^{1}$-norm without harmonicity. Then we can follow the arguments in $[1,7,8]$.

\section{JACOBi Field Estimate}

We take $K_{r_{0}}$ in Theorem 1.1 such that $K_{r_{0}} / r_{0}<1 / 100$ and $K_{r_{0}}<1 / 100$. From now on, $\|\cdot\|$ is the norm on Euclidean space. Let $\psi=\phi^{-1}=\left(u_{1}, u_{2}, \cdots, u_{n}\right)$ be a coordinate map such that $\phi$ satisfies the properties in Definition 2.1. Let $p=\phi(O)$. We may assume that $B\left(p, \frac{r_{0}}{15}\right) \subset\left(B\left(0, r_{0}\right)\right)$. Let $B_{\phi}\left(O, \frac{n}{15}\right)$ be the set $B\left(O, \frac{r_{0}}{15}\right) \subset B\left(0, r_{0}\right)$ with the metric $\phi^{*} g$. Let $\gamma$ be a unit speed geodesic in $B_{\phi}\left(O, \frac{r_{0}}{15}\right)$ with $\gamma(0)=O$. Let $r(x)$ be the distance $d(O, x)$ from $O$ to $x$ with respect to $\phi^{*} g$. We have

$$
\begin{aligned}
0 & =2\left\langle\nabla_{\gamma^{\prime}(t)} \gamma^{\prime}(t), \frac{\partial}{\partial u_{i}}\right\rangle=2 \frac{d}{d t}\left\langle\gamma^{\prime}(t), \frac{\partial}{\partial u_{i}}\right\rangle-\frac{\partial}{\partial u_{i}}\langle\nabla r, \nabla r\rangle \\
& =2 \frac{d}{d t}\left\langle\gamma^{\prime}(t), \frac{\partial}{\partial u_{i}}\right\rangle .
\end{aligned}
$$

Then we obtain that $\left\langle\gamma^{\prime}(t), \frac{\partial}{\partial u_{i}}\right\rangle=V_{i}$ for some constant $V_{i}$. We denote by $V$ the vector $\left(V_{1}, V_{2}, \cdots, V_{n}\right)$. Let $\psi_{*}\left(\gamma^{\prime}(t)\right)=\left(v_{1}(t), v_{2}(t), \cdots, v_{n}(t)\right)$ and $g_{i j}(=$ $\left.g_{\tau, i j}\right)=\left\langle\frac{\partial}{\partial u_{i}}, \frac{\partial}{\partial u_{j}}\right\rangle$. Then we have

$$
\sum_{j} v_{j} g_{i j}=V_{i}
$$

which implies that

$$
\psi_{*}\left(\gamma^{\prime}(t)\right)=v(t)=\bar{g}(\gamma(t)) V
$$

for $\bar{g}=\left(g_{i j}\right)^{-1}$. Note that we may assume that $g_{i j}(O)=\delta_{i j}$ by changing coordinate functions slightly. Precisely, let $\sum_{j} a_{i j} u_{j}=w_{i}$, where $a_{i j}$ is a constant. Then

$$
\frac{\partial}{\partial u_{i}}=\sum_{l} a_{l i} \frac{\partial}{\partial w_{l}} .
$$


If $\tilde{g}_{i j}=\left\langle\frac{\partial}{\partial w_{i}}, \frac{\partial}{\partial w_{j}}\right\rangle$, then

$$
g_{i j}=\sum_{l, k} a_{l i} \tilde{g}_{l k} a_{k j}
$$

If we let $A=\left(a_{i j}\right)$, then

$$
g=A^{t} \tilde{g} A
$$

where $A^{t}$ is the transpose of $A$. Hence, if we take $A$ such that $g(O)=A^{t} A$, then $\tilde{g}(O)=\delta$. Since $g$ is a positive definite symmetric matrix, there exists an orthogonal matrix $P$ such that $P^{t} g(O) P$ is a diagonal matrix $T$ whose diagonal entries are $\lambda_{1}, \cdots, \lambda_{n}>0$. We have that $g(O)=A^{t} A$ if and only if

$$
A=\sqrt{T} P^{t},
$$

where $\sqrt{T}$ is a diagonal matrix whose diagonal entries are $\sqrt{\lambda_{i}}$, s. Since $K_{r_{0}}<$ $1 / 100$,

$$
e^{-\frac{1}{100}} \leq \min _{i} \sqrt{\lambda_{i}} \leq\|A\| \leq \max _{i} \sqrt{\lambda_{i}} \leq e^{\frac{1}{100}} .
$$

So if we take $\left\{w_{i}\right\}$ as coordinate functions, from (3.2), we obtain that

$$
\begin{gathered}
e^{-\frac{1}{25}} \delta \leq \tilde{g} \leq e^{\frac{1}{25}} \delta \\
\|\partial \tilde{g}\|_{C^{0}} \leq e^{\frac{1}{50}}\|\partial g\|_{C^{0}} \leq \frac{1}{100} e^{\frac{1}{50}}<\frac{1}{50} .
\end{gathered}
$$

From now on, we will abbreviate $\tilde{g}$ to $g$. Hence we may assume that $g_{i j}(O)=\delta_{i j}$ and

$$
\psi_{*}\left(\gamma^{\prime}(t)\right)=v(t)=\bar{g}(\gamma(t)) v(0) .
$$

If $x, y \in B_{\phi}\left(O, \frac{r_{0}}{15}\right)$ then for the new coordinate map $\psi=\left(w_{1}, w_{2}, \cdots, w_{n}\right)$,

$$
e^{-\frac{1}{25}}\|\psi(x)-\psi(y)\| \leq d(x, y) \leq e^{\frac{1}{25}}\|\psi(x)-\psi(y)\| .
$$

Let $Q(t, \theta)=\psi(\exp t \theta)$ and $G(t, \theta)=\bar{g}(Q(t, \theta))$. Then (3.5) implies that

$$
\frac{\partial Q}{\partial t}(t, \theta)=G(t, \theta) \frac{\partial Q}{\partial t}(0, \theta) .
$$

So

$$
\frac{\partial^{2} Q}{\partial \theta \partial t}(t, \theta)=\frac{\partial G}{\partial \theta}(t, \theta) \frac{\partial Q}{\partial t}(0, \theta)+G(t, \theta) \frac{\partial^{2} Q}{\partial \theta \partial t}(0, \theta)
$$

Then

$$
\frac{\partial Q}{\partial \theta}\left(t, \theta_{0}\right)=\int_{0}^{t}\left(\frac{\partial G}{\partial \theta}\left(u, \theta_{0}\right) \frac{\partial Q}{\partial t}\left(0, \theta_{0}\right)+G\left(u, \theta_{0}\right) \frac{\partial^{2} Q}{\partial \theta \partial t}\left(0, \theta_{0}\right)\right) d u
$$


Since $\bar{g} g=I$, we have $\partial \bar{g}=-\bar{g}(\partial g) g^{-1}$. So if $e^{-\frac{1}{25} \delta} \leq g \leq e^{\frac{1}{25}} \delta$ and $\|\partial g\|_{C^{0}} \leq$ $\frac{1}{100} e^{\frac{1}{50}}$, then $\|\partial \bar{g}\|_{C^{0}} \leq \frac{1}{50}$. From (3.5), we have

$$
\frac{\left\|G\left(t_{1}, \theta_{1}\right)-G\left(t_{2}, \theta_{2}\right)\right\|}{\left\|Q\left(t_{1}, \theta_{1}\right)-Q\left(t_{2}, \theta_{2}\right)\right\|} \leq \frac{2}{3} K_{1}
$$

so from (3.7),

$$
\left\|\frac{\partial G}{\partial \theta}\right\| \leq \frac{2}{3} K_{1}\left\|\frac{\partial Q}{\partial \theta}\right\|
$$

for $t<\frac{r_{0}}{15}$ and some constant $K_{1}>0$. It should be noted that

$$
\frac{2}{3} K_{1}<2 \frac{K_{r_{0}}}{r_{0}}<\frac{1}{50} .
$$

Also we have $e^{-\frac{1}{25}} \leq\left\|\frac{\partial Q}{\partial t}\left(0, \theta_{0}\right)\right\| \leq e^{\frac{1}{25}}$ from (3.3) and

$$
\left\|G\left(t, \theta_{0}\right) \frac{\partial^{2} Q}{\partial \theta \partial t}\left(0, \theta_{0}\right)\right\| \leq\left\|G\left(t, \theta_{0}\right)\right\|\left\|\frac{\partial^{2} Q}{\partial \theta \partial t}\left(0, \theta_{0}\right)\right\| \leq e^{\frac{1}{10}}
$$

since

$$
\left\|\frac{\partial^{2} Q}{\partial \theta \partial t}\left(0, \theta_{0}\right)\right\|=\lim _{h \rightarrow 0}\left\|\psi_{*}\left(\frac{\exp _{*}\left(\frac{\partial}{\partial t}\right)\left(0, \theta_{0}+h\right)-\exp _{*}\left(\frac{\partial}{\partial t}\right)\left(0, \theta_{0}\right)}{h}\right)\right\| \leq e^{\frac{1}{25}} .
$$

Then (3.6) implies that

$$
\left\|\frac{\partial Q}{\partial \theta}\left(t, \theta_{0}\right)\right\| \leq K_{1} \int_{0}^{t}\left\|\frac{\partial Q}{\partial \theta}\left(u, \theta_{0}\right)\right\| d u+e^{\frac{1}{10}} t .
$$

By the same reason as above, we have

$$
\left\|G\left(t, \theta_{0}\right) \frac{\partial^{2} Q}{\partial \theta \partial t}\left(0, \theta_{0}\right)\right\|>e^{-\frac{1}{10}}
$$

Then we obtain that

$$
\left\|\frac{\partial Q}{\partial \theta}\left(t, \theta_{0}\right)\right\| \geq-K_{1} \int_{0}^{t}\left\|\frac{\partial Q}{\partial \theta}\left(u, \theta_{0}\right)\right\| d u+e^{-\frac{1}{10}} t .
$$

Let $K_{2}, K_{3}$ be $e^{\frac{1}{10}}, e^{-\frac{1}{10}}$, respectively. We define a function $f$ as follows:

$$
f(t)=\int_{0}^{t}\left\|\frac{\partial Q}{\partial \theta}\left(u, \theta_{0}\right)\right\| d u .
$$

Then the above inequalities imply that

$$
K_{3} t-K_{1} f \leq f^{\prime} \leq K_{2} t+K_{1} f .
$$


Multiplying $e^{-K_{1} t}$ in the second inequality of (3.11), we obtain

$$
\left(e^{-K_{1} t} f\right)^{\prime}=e^{-K_{1} t} f^{\prime}-K_{1} e^{-K_{1} t} f \leq K_{2} t e^{-K_{1} t} .
$$

Integrating both sides with the initial condition $f(0)=0$ and multiplying $e^{K_{1} t}$, we have

$$
f(t) \leq K_{2}\left(\frac{e^{K_{1} t}-1-K_{1} t}{K_{1}^{2}}\right) .
$$

We have that $\lim _{x \rightarrow 0} \frac{e^{x}-x-1}{x^{2}}=1 / 2$ and if $x \leq 1 / 50$

$$
\frac{e^{x}-x-1}{x^{2}} \leq 1
$$

For $t \leq \frac{r_{0}}{15}$, we have $K_{1} t \leq K_{1} \frac{r_{0}}{15} \leq K \frac{r_{0}}{5} \leq \frac{1}{500}$ by (3.8), we have

$$
\frac{e^{K_{1} t}-1-K_{1} t}{K_{1}^{2}}=\frac{e^{K_{1} t}-1-K_{1} t}{K_{1}^{2} t^{2}} t^{2} \leq t^{2} .
$$

So we have

$$
\int_{0}^{t}\left\|\frac{\partial Q}{\partial \theta}\left(u, \theta_{0}\right)\right\| d u \leq K_{2} t^{2} .
$$

From (3.9), we obtain that for $t<\frac{r_{0}}{15}$

$$
\begin{aligned}
\left\|\frac{\partial Q}{\partial \theta}\left(t, \theta_{0}\right)\right\| & \leq K_{1} \int_{0}^{t}\left\|\frac{\partial Q}{\partial \theta}\left(u, \theta_{0}\right)\right\| d u+K_{2} t \\
& \leq K_{2}\left(K_{1} t+1\right) t \leq K_{2}\left(\frac{K_{1}}{4} r_{0}+1\right) t \leq L_{1} t
\end{aligned}
$$

for some constant $L_{1} \in\left[\frac{4}{5}, \frac{6}{5}\right]$. From the first inequality of (3.11), we obtain that

$$
\begin{aligned}
\left\|\frac{\partial Q}{\partial \theta}\left(t, \theta_{0}\right)\right\| & \geq K_{3} t-K_{1} \int_{0}^{t}\left\|\frac{\partial Q}{\partial \theta}\left(t, \theta_{0}\right)\right\| d t \\
& \geq K_{3}\left(1-K_{1} t\right) t \geq L_{2} t
\end{aligned}
$$

for some constant $L_{2} \in\left[\frac{4}{5}, \frac{6}{5}\right]$ by the same reason as (3.13).

From (3.13) and (3.14), we have the following lemma:

Lemma 3.1. Let $K_{r_{0}}$ satisfy that $K_{r_{0}}<\frac{1}{100}$ and $\frac{K_{r_{0}}}{r_{0}}<\frac{1}{100}$. Let $\gamma(t)$ be a minimal geodesic starting from $q$ and $J(t)$ is a Jacobi field along $\gamma$ such that $J(0)=0$. Then $\frac{L_{2} t}{s}\|J(s)\| \leq\|J(t)\| \leq \frac{L_{1} t}{s}\|J(s)\|$ if $t<s<\frac{r_{0}}{15}$. 
Proof. In case that $\left\langle J^{\prime}(0), \gamma^{\prime}(0)\right\rangle=0$, we can consider $J$ as $c \frac{\partial Q(t, \theta)}{\partial \theta}$ for a constant $c$, so it is done by (3.13), (3.14). If $\left\langle J^{\prime}(0), \gamma^{\prime}(0)\right\rangle \neq 0$, we can decompose $J$ into tangential and perpendicular components and obtain the lemma, since tangential component is linear in $t$.

\section{Volume Comparison and Proof of Theorems}

We obtain that volumes of $\frac{r_{0}}{15}$-balls in $\tilde{M}$ are bounded above by a constant $v\left(n, r_{0}\right)>0$ depending only on $n, r_{0}$ by (3.13). Let $S_{x}$ be the maximal $r_{0} / 100$ separated set in $B\left(x, \frac{r_{0}}{15}\right)$. Then $\bigcup_{S_{x}} B\left(x, r_{0} / 100\right)$ covers $B\left(x, \frac{r_{0}}{15}\right)$.

Around $x$, use exponential polar coordinates and write the volume element as $d \mathrm{vol}=\omega d t \wedge d \theta$ inside the cut locus, where $d \theta$ is the standard volume element on the unit sphere. Outside the cut locus, $\omega=0$. From Lemma 3.1, for $t<r_{0} / 300$,

$$
\omega(t, \theta) \geq\left(\frac{300 L_{2} t}{r_{0}}\right)^{n-1} \omega\left(\frac{r_{0}}{300}, \theta\right)
$$

and for $\frac{r_{0}}{15}>s>r_{0} / 300$,

$$
\omega(s, \theta) \leq\left(\frac{300 s}{L_{2} r_{0}}\right)^{n-1} \omega\left(\frac{r_{0}}{300}, \theta\right) .
$$

Then

$$
\operatorname{vol}\left(B\left(x, \frac{r_{0}}{300}\right)\right) \geq D_{1}\left(n, r_{0}\right) \int_{S^{n-1}} \omega\left(\frac{r_{0}}{300}, \theta\right) d \theta
$$

and

$$
\operatorname{vol}\left(B\left(x, \frac{r_{0}}{15}\right)\right) \leq \operatorname{vol}\left(B\left(x, \frac{r_{0}}{300}\right)\right)+D_{2}\left(n, r_{0}\right) \int_{S^{n-1}} \omega\left(\frac{r_{0}}{300}, \theta\right) d \theta
$$

for some constant $D_{1}, D_{2}$ depending only on $n, r_{0}$. So

$$
\frac{\operatorname{vol}\left(B\left(x, \frac{r_{0}}{15}\right)\right)}{\operatorname{vol}\left(B\left(x, \frac{r_{0}}{300}\right)\right)} \leq 1+\frac{D_{2}}{D_{1}} .
$$

Remark 4.1. One may consider that the volume comparison (4.1) is obtained only with a weak $C^{0}$-norm bound. If $\phi=\exp$, then the domain of $\phi$ inside the cut locus for $\tilde{M}$ is star-shaped, so we can obtain (4.1) with a weak $C^{0}$-norm bound. But in general, the non overlapping domain of a weak coordinate need not be starshaped, so we cannot obtain (4.1) only with a weak $C^{0}$-norm bound.

Let $S$ be the maximal $r_{0} / 100$-separated set of $\tilde{M}$. We make a graph $G$ by adding edges between points $x, y$ if $d(x, y) \leq \frac{r_{0}}{40}$. If we let the length of each edge be 1 , then $G$ is a length space. Then we have a metric $d_{S}$ on $S$ induced from $G$. 
Lemma 4.2. $\frac{r_{0}}{300} d_{S}(x, y) \leq d(x, y) \leq \frac{r_{0}}{40} d_{S}(x, y)$ for $x, y \in S$.

Proof. Let $\gamma:[0, L] \rightarrow \tilde{M}$ be a normal length minimizing geodesic from $x$ to $y$. Let $\left\{t_{i} \mid t_{0}=0, t_{N}=L, t_{i}<t_{i+1}\right\}$ be a partition of $[0, L]$ such that $t_{i+1}-t_{i}=\frac{r_{0}}{200}$ for $i+1<N$. Then $N \leqq \frac{200 L}{r_{0}}+1$. For each $\gamma\left(t_{i}\right)$, there exists $x_{i} \in S$ such that $d\left(\gamma\left(t_{i}\right), x_{i}\right) \leq r_{0} / 100$. Then $d\left(x_{i}, x_{i+1}\right) \leq \frac{r_{0}}{40}$ and $d_{S}(x, y) \leq \frac{200 L}{r_{0}}+1=$ $\frac{200 d(x, y)}{r_{0}}+1$. Since $d(x, y) \geq \frac{r_{0}}{100}$ for $x, y \in S$, we obtain

$$
d_{S}(x, y) \leq\left(\frac{200}{r_{0}}+\frac{1}{d(x, y)}\right) d(x, y) \leq \frac{300}{r_{0}} d(x, y)
$$

Conversely, if $d_{S}(x, y)=m$, then there exists $x_{0}=x, x_{1}, \cdots, x_{m}=y \in S$ such that $d\left(x_{i}, x_{i+1}\right) \leq \frac{r_{0}}{40}$. Then

$$
d(x, y) \leq \sum_{i=0}^{m} d\left(x_{i}, x_{i+1}\right) \leq \frac{r_{0}}{40} m \leq \frac{r_{0}}{40} d_{S}(x, y) .
$$

So we obtain the lemma.

From the above lemma, we obtain that $B(x, R) \subset B_{d_{S}}\left(x, 300 R / r_{0}\right)$ for $x \in S$. Since $B\left(y, r_{0} / 300\right)$ 's for $y \in S \cap B\left(x, \frac{r_{0}}{40}\right)$ are disjoint in $B\left(x, \frac{r_{0}}{15}\right)$, we have

$$
\left|B_{d_{S}}(x, 1)\right| \leq \frac{\operatorname{vol}\left(B\left(y_{0}, \frac{r_{0}}{15}\right)\right)}{\operatorname{vol}\left(B\left(y_{0}, \frac{r_{0}}{300}\right)\right)} \leq H \frac{D_{2}}{D_{1}}
$$

for $x \in S$ and $\operatorname{vol}\left(B\left(y_{0}, \frac{r_{0}}{300}\right)\right)=\min _{y \in B\left(x, \frac{r_{0}}{40}\right)} \operatorname{vol}\left(B\left(y, \frac{r_{0}}{300}\right)\right)$. If we let $N$ be $1+\frac{D_{2}}{D_{1}}$, then we obtain that

$$
\left|B_{d_{S}}\left(x, 300 R / r_{0}\right)\right| \leq N^{300 R / r_{0}} .
$$

Then we obtain the following volume growth

$$
\operatorname{vol}(B(x, R)) \leq N^{300 R / r_{0}} v\left(n, r_{0}\right) .
$$

From (4.2), we have

$$
\left|B_{d_{S}}\left(x, 3000 D / r_{0}\right)\right| \leq N^{3000 D / r_{0}} .
$$

Then we obtain that

$$
\operatorname{vol}(B(x, 10 D)) \leq N^{3000 D / r_{0}} v\left(n, r_{0}\right) .
$$


We may assume that $D>\frac{r_{0}}{15}$. Let $S^{\prime}$ be the maximal $4 D$-separated subset of $\tilde{M}$ and $S^{\prime}(R)=\left\{x \in S^{\prime} \mid d(q, x) \leq R\right\}$. Then for each $x_{i} \in S^{\prime}(R+2 D) \backslash S^{\prime}(R-4 D)$, we have

$$
B(q, R+2 D) \subset \bigcup_{x_{i} \in S^{\prime}(R-4 D)} B\left(x_{i}, 10 D\right)
$$

since there exists $x_{j} \in S^{\prime}(R-4 D)$ for each $x_{i} \in S^{\prime}(R+2 D) \backslash S^{\prime}(R-4 D)$ such that $d\left(x_{i}, x_{j}\right) \leq 10 D$. Also we have

$$
B(q, R) \supset \bigcup_{x_{i} \in S^{\prime}(R-4 D)} B\left(x_{i}, 2 D\right) .
$$

Then

$$
\begin{aligned}
\operatorname{vol}(B(q, R+2 D)) & \leq \sum_{x_{i} \in S^{\prime}(R-4 D)} \operatorname{vol}\left(B\left(x_{i}, 10 D\right)\right) \\
& \leq\left|S^{\prime}(R-4 D)\right| N^{3000 D / r_{0}} v\left(n, r_{0}\right) .
\end{aligned}
$$

Since every $2 D$-ball contains a fundamental domain, we have $\operatorname{vol}\left(B\left(x_{i}, 2 D\right)\right) \geq v$. Since $\cup_{x_{i} \in S^{\prime}(R-4 D)} B\left(x_{i}, 2 D\right)$ is a disjoint union in $B(q, R)$, we obtain that

$$
\operatorname{vol}(B(q, R)) \geq\left|S^{\prime}(R-4 D)\right| v .
$$

Hence we obtain from (2.3) that

$$
\begin{aligned}
\frac{k_{q}(\lambda, p, R)}{\operatorname{vol}(B(q, R))} & \leq \frac{\operatorname{vol}(B(q, R+2 D))}{\operatorname{vol}(B(q, R))} \bar{k}(\lambda, p) \\
& \leq \frac{N^{3000 D / r_{0}} v\left(n, r_{0}\right)}{v} \bar{k}(\lambda, p),
\end{aligned}
$$

which completes the proof of Theorem 1.1 with Theorem 2.2.

Proof of Theorem 1.2(a). For any $\beta<1$, there exists an $\epsilon_{1}\left(n, p, \beta, v, D, r_{0}, C_{0}, R\right)>$ 0 such that if

$$
\bar{k}(\lambda, p)<\epsilon_{1}
$$

then for $r<R$,

$$
\beta \frac{v_{\lambda}(n, r)}{v_{\lambda}(n, R)} \leq \frac{\operatorname{vol}(B(x, r))}{\operatorname{vol}(B(x, R))}
$$

from (4.4) and (2.2). To follow Anderson's arguments [1], we need a (relative) volume comparison for $3 D$-balls in $\tilde{M}$. From (4.6), if we take $\beta$ and $R$ to be $9 / 10$ and $3 D$, respectively, we can prove Theorem 1.2(a). Furthermore, with Theorem 
1.2(a) and the arguments in [7], we obtain that $\pi_{1}(M)$ has a polynomial growth of rank $\leq n$.

Proof of Theorem 1.2(b). In [5], they obtain the following splitting theorem:

Theorem 4.3. Let $\left(M_{k}, x_{k}\right)$ be a sequence of pointed complete Riemannian manifolds which converge to a limit space $(X, x)$ in the pointed Gromov-Hausdorff topology. If $\left(M_{k}, x_{k}\right)$ satisfies that for any $p>n / 2$,

$$
\begin{aligned}
& \operatorname{vol}\left(B\left(x_{k}, 1\right)\right) \geq v>0, \\
& \sup _{x \in M_{k}} \frac{k_{x}(0, p, R)}{\operatorname{vol}(B(x, R))} \rightarrow 0
\end{aligned}
$$

as $k \rightarrow \infty$, then the spitting theorem holds for $X$, i.e. if $X$ contains a line, then $X$ is isometric to $\mathbb{R} \times X^{\prime}$.

From the above volume comparison (4.6) for $\tilde{M}$ and the convergence theorem in [2], we can prove that if a sequence $M_{k}$ satisfies the conditions in Theorem 1.2 and $k(0, p)$ for $M_{k}$ converges to 0 , then $\left(\tilde{M}_{k}, x_{k}\right)$ converges to a limit space $(X, x)$. If $X$ contains a line, then $X$ is isometric to $\mathbb{R} \times X^{\prime}$ by (4.4) and Theorem 4.3. Then there are no obstruction to follow the arguments in [8], which completes the proof of Theorem 1.2(b).

Proof of Theorem 1.3. We may assume that $r_{0} \leq 1$. If $\|(M, g)\|_{C^{1, \alpha}, r_{0}} \leq k$, then

$$
\frac{\|g(x)-g(y)\|}{\|x-y\|} \leq \frac{k}{r_{0}^{1+\alpha}}\|x-y\|^{\alpha} \leq \frac{k}{r_{0}^{1+\alpha}} r^{\alpha}
$$

for $x, y \in B\left(p, \frac{r_{0}}{15}\right)$. So there exists an $r_{1}>0$ such that $\|\partial g\|_{C^{0}} \leq 1 / 100$ on $B\left(p, r_{1}\right)$.

We take new weak coordinates such that $\tilde{g}(O)=I$ as we see in Section 3. Since $\tilde{g}=A g A^{t}$ for a constant matrix $A=\sqrt{T} P^{t}$ and $P \in O(n, \mathbb{R})$, we also obtain that

$$
\|\tilde{g}(x)-\tilde{g}(O)\| \leq\|\partial g\|_{C^{0}}\|T \mid\|\|x\|
$$

since $P \in O(n, \mathbb{R})$. Since $T$ is the diagonal matrix whose diagonal entries are eigenvalues of $g$. Since $\|g\| \leq e^{2 k} \delta, \tilde{g}(x) \rightarrow \delta=\tilde{g}(O)$ uniformly as $\|x\| \rightarrow 0$. So there exists $r_{2}>0$ such that $e^{-\frac{1}{100} \delta} \leq g \leq e^{\frac{1}{100}} \delta$ on $B\left(p, r_{2}\right)$. Hence if we use $\tilde{r}_{0}=\min \left(r_{1}, r_{2}\right)$ instead of $r_{0}$ in the proof of Theorem 1.1 and Theorem 1.2, we can prove Corollary 1.3.

\section{REFERENCES}

1. M. Anderson, Short geodesics and gravitational instantons, J. Diff. Geom., 31 (1990), 265-275. 
2. M. Gromov, Metric structures for Riemannian and non-Riemannian spaces, Birkhäuser, 1998.

3. S.-H. Paeng, Topological entropy for geodesic flows under a Ricci curvature condition, Proc. Amer. Math. Soc., 125 (1997), 1873-1879.

4. P. Petersen, G. Wei, Relative volume comparison with integral curvature bounds, GAFA, 7 (1997), 1031-1045.

5. P. Petersen, G. Wei, Geometry and analysis on manifolds with integral Ricci curvature bounds II, Trans. Amer. Math. Soc., 353 (2000), 457-478.

6. P. Petersen, G. Wei, R. Ye, Controlled geometry via smoothing, Comment. Math. Helv, 74 (1999), 345-363.

7. G. Wei, On the fundamental group of manifolds with almost nonnegative Ricci curvature, Proc. Amer. Math. Soc., 110 (1990), 197-199.

8. G.-J. Yun, A note on the fundamental groups of almost non-negative curvature manifolds, Proc. Amer. Math. Soc., 125 (1997), 1517-1522.

9. J.-G. Yun, On the fundamental groups of manifolds with integral curvature bounds, Arch. Math., 85 (2005), 167-174.

\section{Seong-Hun Paeng}

Department of Mathematics,

Konkuk University,

1 Hwayang-dong, Gwangjin-gu,

Seoul 143-701,

Korea

E-mail: shpaeng@konkuk.ac.kr 